\title{
Clinical significance of overexpression of NRG1 and its receptors, HER3 and HER4, in gastric cancer patients
}

\author{
Sumi Yun ${ }^{1,5} \cdot$ Jiwon Koh $^{1} \cdot$ Soo Kyung $\mathrm{Nam}^{2} \cdot \mathrm{Jung}_{\text {Ok Park }}^{2} \cdot$ Sung Mi Lee ${ }^{2}$. \\ Kyoungyul Lee ${ }^{3} \cdot \mathrm{Kyu}$ Sang Lee ${ }^{2} \cdot$ Sang-Hoon $\mathrm{Ahn}^{4} \cdot$ Do Joong Park ${ }^{4} \cdot$ \\ Hyung-Ho Kim ${ }^{4}$ - Gheeyoung Choe ${ }^{2}$ Woo Ho Kim ${ }^{1} \cdot$ Hye Seung Lee ${ }^{2}$
}

Received: 7 March 2017/ Accepted: 25 May 2017 / Published online: 1 June 2017

(C) The International Gastric Cancer Association and The Japanese Gastric Cancer Association 2017

\begin{abstract}
Background Neuregulin 1 (NRG1), a ligand for human epidermal growth factor (HER) 3 and HER4, can activates cell signaling pathways to promote carcinogenesis and metastasis.

Methods To investigate the clinicopathologic significance of NRG1 and its receptors, immunohistochemistry was performed for NRG1, HER3, and HER4 in 502 consecutive gastric cancers (GCs). Furthermore, HER2, microsatellite instability (MSI), and Epstein-Barr virus (EBV) status were investigated. NRG1 gene copy number (GCN) was determined by dual-color fluorescence in situ hybridization (FISH) in 388 available GCs.

Results NRG1 overexpression was observed in 141 (28.1\%) GCs and closely correlated with HER3 $(P=0.034)$ and HER4 $(P<0.001)$ expression. NRG1 overexpression was significantly associated with
\end{abstract}

Electronic supplementary material The online version of this article (doi:10.1007/s10120-017-0732-7) contains supplementary material, which is available to authorized users.

Hye Seung Lee

hye2@snu.ac.kr

1 Department of Pathology, Seoul National University College of Medicine, Seoul, South Korea

2 Department of Pathology, Seoul National University Bundang Hospital, 173-82 Gumi-ro, Bundang-gu, Seongnam-si, Gyeonggi-do 13620, South Korea

3 Department of Pathology, Kangwon National University Hospital, Chuncheon, Kangwon, South Korea

4 Department of Surgery, Seoul National University Bundang Hospital, Seongnam, South Korea

5 Department of Diagnostic Pathology, Samkwang Medical Laboratories, Seoul, South Korea aggressive features, including infiltrative tumor growth, lymphovascular, and neural invasion, high pathologic stage, and poor prognosis (all $P<0.05$ ), but not associated with EBV, MSI, or HER2 status. Multivariate analysis identified NRG1 overexpression as an independent prognostic factor for survival $(P=0.040)$. HER3 and HER4 expressions were observed in $157(31.3 \%)$ and 277 $(55.2 \%)$, respectively. In contrast to NRG1, expression of these proteins was not associated with survival. NRG1 GCN gain $(\mathrm{GCN} \geq 2.5)$ was detected in $14.7 \%$ patients, including two cases of amplification, and was moderately correlated with NRG1 overexpression ( $\kappa, 0.459$; $P<0.001)$.

Conclusions Although our results indicate a lack of prognostic significance of HER3 and HER4 overexpression in GC, overexpression of their ligand, NRG1, was associated with aggressive clinical features and represented an independent unfavorable prognostic factor. Therefore, NRG1 is a potential prognostic and therapeutic biomarker in GC patients.

Keywords Gastric cancer · Neuregulin 1 . Immunohistochemistry · Fluorescence in situ hybridization · Copy number gain

\section{Introduction}

Despite recent diagnostic and therapeutic advances, gastric cancer (GC) remains a leading cause of cancer deaths, particularly in South Korea [1]. Deeper understanding of the molecular pathogenesis of GC has contributed to successful clinical application of targeted drugs, for example, drugs targeting to human epidermal growth factor receptor (HER) 2 mutations [2]. The HER family consists of four 
transmembrane proteins, HER1 (EGFR), HER2, HER3, and HER4. HER2 is well studied and can induce cell proliferation, differentiation, and apoptosis [2]. HER2 overexpression has been found in a subset (20-30\%) of GC samples, primarily as a result of $H E R 2$ gene amplification $[2,3]$, and currently, drugs targeting HER2-positive GC are increasingly used as part of treatment for patients with advanced GC, as they can significantly improve outcomes $[3,4]$. Unfortunately, a significant number of these patients eventually develop drug resistance and exhibit poor survival rates [4, 5]; hence, recent studies have focused on other members of the HER family, including HER3 and HER4 and their ligands.

Neuregulin (NRG) is a ligand of HER family protein, which has more than 32 isoforms. NRG1 is the predominant ligand of HER3 and HER4. Through binding to HER3, it functions in specific regulation of cell proliferation and organ development [6,7]. Additionally, NRG1 can induce carcinoma development and promote metastasis [7]. Interestingly, recent studies have suggested that PI3K/Akt activation through the NRG1/HER3 signaling pathway leads to development of resistance to HER2-targeted treatment, and it has been proposed that inhibition of this signaling pathway has potential as a therapeutic option to overcome resistance to anti-HER2 treatment [8-11]. However, few studies have assessed the association of NRG1 status and GC or the clinicopathologic significance of the NRG1/HER3/HER2 and NRG1/HER4/HER2 axis in GC.

Unlike other HER family proteins, HER3 lacks significant tyrosine kinase activity; it has a regulatory function through heterodimer formation with other members of the HER family [12]. Heterodimer containing HER3 can activate the following two key signaling pathways: mitogen-activated protein kinase (MAPK) and phosphoinositide 3-kinase (PI3K)/Akt [12]. In various cancers, HER3/ HER2/PI3K/Akt signaling promotes tumor cell proliferation and survival $[6,12,13]$. Several studies have demonstrated associations between HER3 protein expression and poor survival in various cancers including GC [14-17].

HER4 has markedly different functions in tumors, including functionally distinct splice isoforms and multiple proteolytically derived types. Alternative splicing of HER4 releases its intracellular domain and enables it to translocate to the nucleus [18-20]. Although the function of nuclear HER4 has not been fully elucidated, it has a role as a transcriptional cofactor [19]. Several previous studies have reported various prognostic associations with HER4 immunohistochemistry (IHC) results, particularly in breast cancer, including a correlation between cytoplasmic HER4 and improved prognosis [18]. However, the prognostic role of cytoplasmic and nuclear expression of HER4 in GC remains unclear. Moreover, detailed information regarding the mechanism of action of HER4 and its relationship with its ligand in gastric cancer is lacking [17].

In this study, we aimed to determine the prevalence and clinicopathologic implications of NRG1 expression in a large cohort of GC samples and to assess the relationship between NRG1 expression and that of HER3 and HER4. In addition, NRG1 expression status in GC was compared with HER2 positivity, Epstein-Barr virus (EBV) in situ hybridization (ISH), and microsatellite instability (MSI) status. We evaluated the NRG1 gene copy number (GCN) status using dual-color fluorescence in situ hybridization (FISH) analysis and compared the concordance rate between protein expression and genetic alteration for NRG1.

\section{Materials and methods}

\section{Patients and clinicopathologic characteristics}

A total of 502 consecutive GC patients who had curative surgery at Seoul National University Bundang Hospital from May 2003 to December 2005 were analyzed in this study. Clinical information including age, sex, size, location, and pathologic stage were collected retrospectively from medical records retrospectively. Patients who had received preoperative chemotherapy or radiotherapy were excluded from this study. The American Joint Committee on Cancer seventh staging system was used to determine pTNM stage [21]. Disease-specific survival (DSS) data were collected, including patient outcome, the interval between the date of surgery and the date of death due to $\mathrm{GC}$, and the period of disease-free survival (DFS) from surgery until the date of disease progression, death, or last disease assessment.

\section{Tissue microarray (TMA) construction}

TMA blocks were constructed using previously described methods [22]. Briefly, we selected a representative tumor area for TMA construction in each case, and tissue cores of $2 \mathrm{~mm}$ diameter were transferred to the TMA block. Samples were considered valid when the tumor occupied more than $15 \%$ of the core area. Serial sections were cut and used for IHC and FISH analyses.

\section{Immunohistochemistry}

We performed IHC using anti-NRG1 (1:2000, Abcam, Cambridge, MA, USA), anti-HER3 (1:3000, Thermo Scientific, Fremont, CA, USA), anti-HER4 (1:8000, Thermo scientific), and anti-HER2 (4B5; pre-dilution; Ventana 
Medical Systems, Tucson, AZ, USA) antibodies with a Ventana Benchmark automatic immunostaining system (BenchMark XT, Ventana Medical system), according to the manufacturer's instructions. Antigen retrieval for immunohistochemistry consisted of Cell Conditioning 1 (CC1) (pH 8.4) for $24 \mathrm{~min}$ at $100{ }^{\circ} \mathrm{C}$. Sections on microslides were incubated with these antibodies and immunoreactivity detected using diaminobenzidine (DAB) substrate. Immunostaining was interpreted without prior knowledge of clinicopathologic data. NRG1, HER3, and HER4 were faintly expressed in the foveolar glands of nonneoplastic gastric mucosa; however, weak to moderate expression was observed in the cytoplasm of deep gastric glands. In tumor cells, NRG1 expression was detected in the cytoplasm and HER3 expression in the cytoplasm and/ or membrane of tumor cells. HER4 expression was also observed in the cytoplasm of tumor cells; however, a significant fraction of $\mathrm{GC}$ exhibited nuclear expression of HER4; therefore, we recorded cytoplasmic and nuclear expression of HER4 separately. We evaluated both the extent $(\%)$ and the intensity of positive tumor cells. The intensity of NRG1, HER3, and HER4 protein expression was classified into the following four categories according to the scoring system presented in a previous report [15]: 0 , negative; $1+$, weak positive; $2+$, moderate positive; $3+$, strong positive. For statistical analysis, cases with the immunostaining intensity of $2+$ or $3+$ in $10 \%$ or more tumor cells were defined as positive or overexpression of NRG1 and its receptors.

\section{NRG1 analysis by dual-color fluorescence in situ hybridization}

We performed FISH analysis to evaluate NRG1 GCN. Of the 502 cases, 388 were interpretable by FISH analysis. Samples that were negative for tumor cells or without FISH signals were excluded. NRG1 gene status was evaluated by dual-color FISH assay according to the manufacturer's instructions [23]. TMA slides ( $2 \mu \mathrm{m}$ in thickness) were incubated with a NRG1 probe (Macrogen Inc., Seoul, Korea) and centromeric enumeration probe 8 (CEP8, Macrogen Inc., Seoul, Korea) with pepsin at $37^{\circ} \mathrm{C}$ for $30 \mathrm{~min}$. After being placed in HYBrite solution (Abbott Laboratories, Abbott Park, IL, USA) at $74{ }^{\circ} \mathrm{C}$, slides were counterstained with DAPI (Macrogen, Inc., Seoul, Korea). FISH analysis was evaluated without prior knowledge of clinicopathologic information. Entire cores were scanned and signals in 20 non-overlapping tumor nuclei counted in each core. If clusters were observed, small and large clusters were considered as 6 and 12 signals, respectively. NRG1 amplification was defined as an NRG1/CEP8 ratio of $\geq 2$.0. In addition to NRG1 amplification, increased $N R G 1$ GCN signals were also observed. Since there are no standardized guidelines for evaluation of NRG1 gene status, we used a cutoff value adapted from a previous study on EGFR in gastric cancer [24]; hence, NRG1 GCN gain was defined as the copy number of $N R G 1$ per nucleus of $\geq 2.5$.

\section{Evaluation of HER2 status}

HER2 status was determined according to the results of IHC and silver ISH (SISH), as described previously [25]. Briefly, HER2 protein expression was evaluated according to the DAKO guideline for scoring HercepTest ${ }^{\mathrm{TM}}$ in GC. HER2 gene status was evaluated using a Ventana BenchMark XT device (Ventana Medical Systems). INFORM HER2 DNA and INFORM Chromosome 17 (CEP17) were used for automatic SISH staining. HER2 positivity was indicated when cancer cells had IHC scores of $2+$ or $3+$ in addition to HER 2 gene amplification based on SISH.

\section{Microsatellite instability status}

Tissue sections were obtained from formalin-fixed paraffinembedded blocks, and both tumor and normal areas were microdissected. After deparaffinization with incubation at $70{ }^{\circ} \mathrm{C}$ for $10 \mathrm{~min}$, DNA was extracted using a chelating ion-exchange resin (Instagene matrix; Bio-Rad, Hercules, CA, USA) according to the manufacturer's instructions. MSI analysis was performed using an ABI 3731 automated DNA sequencer (Applied Biosystems, Foster City, CA, USA) with five microsatellite markers (BAT-26, BAT-25, D5S346, D17S250, and D2S123). MSI status was determined into MSI-high (two or more unstable markers), MSIlow (one unstable marker), or microsatellite stable (MSS, no unstable marker) [25].

\section{Epstein-Barr virus in situ hybridization}

EBV ISH using a fluorescein-conjugated EBER oligonucleotide probe (INFORM EBV-encoded RNA probe, Ventana Medical Systems) was performed to determine the EBV status of tumor samples. The cases with cancer cells positive for nuclear EBER were considered EBV-positive GC.

\section{Statistical analyses}

SPSS 21.0 (SPSS Inc., Chicago, IL, USA) was used for statistical analyses. Correlations between NRG1 or HER expression results and clinicopathologic variables were examined using Pearson's chi-square and Fisher's exact tests. The significance of associations with patient outcome was analyzed using Kaplan-Meier survival curves and compared using log rank tests. Univariate and multivariate 
analyses were performed for significant prognostic factors using Cox regression survival analysis. The concordance of NRG1 assessment by IHC and FISH was determined using a Spearman's rank correlation test. Values of $P<0.05$ were considered statistically significant.

\section{Results}

\section{Clinicopathologic characteristics of patients}

The median age of the 502 patients was 62 years (range 25-89 years); $332(66.1 \%)$ were male and 170 (33.9\%) female (Table 1). At the time of surgical treatment, pTNM stages were distributed as follows: $256(51.0 \%)$ cases were at stage I, $78(15.5 \%)$ at stage II, $144(28.7 \%)$ at stage III, and $24(4.8 \%)$ at stage IV. By the Lauren classification, intestinal, diffuse, and mixed type tumors accounted for $217(43.2 \%), 240(47.8 \%)$, and $45(9.0 \%)$ cases, respectively. Of the 502 cases, 239 (47.6\%) had lymph node metastasis. MSI status was evaluated in 489 cases, and 40 $(8.2 \%)$ cases were in the MSI-high group. EBV results were available from $501 \mathrm{GCs}$, among which EBV positivity was observed in $50(10.0 \%)$ cases. NRG1 overexpression was detected in $141(28.1 \%)$ cases. HER3 overexpression was present in 157 (31.3\%) cases, including $13(2.6 \%)$ with membrane staining. Cytoplasmic HER4 expression was observed in $277(55.2 \%)$ cases.

\section{Clinicopathologic significance of NRG1, HER3, and HER4 expression}

The results of analyses of correlations between clinicopathologic variables are presented in Table 1, along with the expression status of NRG1, HER3, and HER4. NRG1 overexpression was more frequently identified in GC with unfavorable clinicopathologic features, including larger tumor size $(P<0.001)$, infiltrative tumor border $(P=0.002)$, vascular invasion $(P=0.012)$, lymphatic invasion $(P<0.001)$, neural invasion $(P<0.001)$, advanced pT stage $(P<0.001)$, lymph node metastasis $(P<0.001)$, and advanced pTNM stage $(P<0.001)$. However, there was no significant correlation between NRG1 positivity by IHC and age, sex, location, or Lauren classification $(P=0.338,0.793,0.244$, and 0.150 , respectively). Among 502 cases, HER3 overexpression correlated strongly with older age $(P<0.001)$ and an expanding tumor border $(P=0.007)$. HER3 overexpression was also more frequently detected in intestinal or mixed type GC than in diffuse type GC $(P<0.001)$ and tended to be detected in tumors located in the lower third of the stomach $(P=0.021)$. HER4 expression did not show any significant association with clinicopathologic characteristics except age $(P=0.011)$ and histologic type by the Lauren criteria $(P=0.007)$. HER2, MSI, and EBV status exhibited no significant correlations with NRG1, HER3, or HER4 expression (all $P>0.05$ ) other than a correlation between HER2 and HER3 $(P=0.022)$.

\section{Survival analysis}

For survival analysis, 501 patients were followed up for 1-109 months, with a median follow-up period of 67 months. The remaining single case was lost to followup after surgery. At the time of analysis, 118 (23.6\%) patients had tumor recurrence and $110(22.0 \%)$ suffered disease-related death. Kaplan-Meier survival analysis revealed that patients with GC overexpressing NRG1 had significantly worse DFS and DSS compared to the NRG1 negative group (both $P<0.001$ ); however, there was no difference in DFS or DSS associated with HER3 or cytoplasmic HER4 overexpression (both $P>0.05$; Fig. 2).

Univariate analysis indicated that NRG1 expression and established prognostic pathologic factors, including tumor size, non-intestinal histology, tumor border, vascular invasion, lymphatic invasion, neural invasion, and pathologic stage, were significantly associated with DFS and DSS. By multivariate analysis, NRG1 overexpression was identified as an unfavorable prognostic factor for DFS (hazard ratio 1.455; 95\% confidence interval 1.009-2.100; $P=0.045$ ) and DSS (hazard ratio 1.490; 95\% confidence interval 1.019-2.177; $P=0.040$ ). Vascular invasion, lymphatic invasion, and pTNM stage were independent prognostic factors for both DFS and DSS. Neural invasion was also independently associated with DSS (Table 2).

\section{Correlation of NRG1 expression status with that of its receptors}

To investigate associations between NRG1 and its receptors, we evaluated the results of NRG1 IHC in comparison with those for HER3 and HER4. As shown in Table 3, there was a close association between NRG1 and HER3 expression $(P=0.034)$ and between NRG1 and cytoplasmic HER4 $(P<0.001)$. HER3 overexpression was significantly related to HER4 cytoplasmic expression $(P<0.001)$.

\section{Evaluation of NRG1 GCN by FISH}

The median NRG1/CEP8 ratio was 1.03 (range 0.57-5.72). Among the available 388 cases, NRG1 GCN gain was detected in $57(14.7 \%)$, including $2(0.5 \%)$ cases of amplification (Fig. 1g, h). When NRG1 GCN status was compared with NRG1 protein expression, $N R G 1$ GCN gain was significantly associated with NRG1 protein expression 
Table 1 The correlation between clinicopathologic parameters and expression status of NRG1, HER3, and HER4

\begin{tabular}{|c|c|c|c|c|c|c|c|c|c|c|}
\hline \multirow[t]{2}{*}{ Characteristics } & \multirow[t]{2}{*}{ Total $(\%)$} & \multicolumn{3}{|l|}{ NRG1 (\%) } & \multicolumn{3}{|l|}{ HER3 (\%) } & \multicolumn{3}{|c|}{ HER4 (cytoplasmic) (\%) } \\
\hline & & Negative & Positive & $P$ & Negative & Positive & $P$ & Negative & Positive & $P$ \\
\hline Total & $502(100.0)$ & 361 (71.9) & $141(28.1)$ & & $345(68.7)$ & $157(31.3)$ & & $225(44.8)$ & $277(55.2)$ & \\
\hline Age (years) & & & & 0.338 & & & $<0.001$ & & & 0.011 \\
\hline$\leq 60$ & $225(44.8)$ & $157(69.8)$ & $68(30.2)$ & & $173(76.9)$ & $52(23.1)$ & & $115(51.1)$ & $110(48.9)$ & \\
\hline$>60$ & $277(55.2)$ & 204 (73.6) & $73(26.4)$ & & $172(62.1)$ & $105(37.9)$ & & $110(39.7)$ & $167(60.3)$ & \\
\hline Sex & & & & 0.793 & & & 0.097 & & & 0.139 \\
\hline Male & $332(66.1)$ & $240(72.3)$ & $92(27.7)$ & & $220(66.3)$ & $112(33.7)$ & & $141(42.5)$ & $191(57.5)$ & \\
\hline Female & $170(33.9)$ & $121(71.2)$ & $49(28.8)$ & & $125(73.5)$ & $45(26.5)$ & & $84(49.4)$ & $86(50.6)$ & \\
\hline Tumor size & & & & $<0.001$ & & & 0.932 & & & 0.726 \\
\hline$\leq 3 \mathrm{~cm}$ & $158(31.5)$ & $131(82.9)$ & $27(17.1)$ & & $109(69.0)$ & $49(31.0)$ & & $69(43.7)$ & $89(56.3)$ & \\
\hline$>3 \mathrm{~cm}$ & $344(68.5)$ & $230(66.9)$ & $114(33.1)$ & & $236(68.6)$ & $108(31.4)$ & & $156(45.3)$ & $188(54.7)$ & \\
\hline Location & & & & 0.244 & & & 0.021 & & & 0.139 \\
\hline Upper third & $80(15.9)$ & $51(63.8)$ & $29(36.3)$ & & $59(73.8)$ & $21(26.3)$ & & $33(41.3)$ & $47(58.8)$ & \\
\hline Middle third & $156(31.1)$ & $119(76.3)$ & $37(23.7)$ & & $118(75.6)$ & $38(24.4)$ & & $82(52.6)$ & $74(47.4)$ & \\
\hline Lower third & $252(50.2)$ & $181(71.8)$ & $71(28.2)$ & & $157(62.3)$ & $95(37.7)$ & & $104(41.3)$ & $148(58.7)$ & \\
\hline Entire & $14(2.8)$ & $10(71.4)$ & 4 (28.6) & & $11(78.6)$ & $3(21.4)$ & & $6(42.9)$ & $8(57.1)$ & \\
\hline Lauren classification & & & & 0.150 & & & $<0.001$ & & & 0.007 \\
\hline Intestinal type & $217(43.2)$ & $164(75.6)$ & $53(24.4)$ & & $122(56.2)$ & $95(43.8)$ & & $82(37.8)$ & $135(62.2)$ & \\
\hline Diffuse type & $240(47.8)$ & 169 (70.4) & $71(29.6)$ & & $196(81.7)$ & $44(18.3)$ & & $125(52.1)$ & $115(47.9)$ & \\
\hline Mixed type & $45(9.0)$ & $28(62.2)$ & $17(37.8)$ & & $27(60.0)$ & $18(40.0)$ & & $18(40.0)$ & $27(60.0)$ & \\
\hline Ming classification & & & & 0.002 & & & 0.005 & & & 0.141 \\
\hline Expanding & $185(36.9)$ & $148(80.0)$ & $37(20.0)$ & & $113(61.1)$ & $72(38.9)$ & & $75(40.5)$ & $110(59.5)$ & \\
\hline Infiltrative & $317(63.1)$ & $213(67.2)$ & $104(32.8)$ & & $232(73.2)$ & $85(26.8)$ & & $150(47.3)$ & $167(52.7)$ & \\
\hline Vascular invasion & & & & 0.012 & & & 0.579 & & & 0.661 \\
\hline Absent & 445 (88.6) & $328(73.7)$ & $117(26.3)$ & & $304(68.3)$ & $141(31.7)$ & & $201(45.2)$ & $244(54.8)$ & \\
\hline Present & $57(11.4)$ & $33(57.9)$ & $24(42.1)$ & & $41(71.9)$ & $16(28.1)$ & & $24(42.1)$ & $33(57.9)$ & \\
\hline Lymphatic invasion & & & & $<0.001$ & & & 0.243 & & & 0.193 \\
\hline Absent & $256(51.0)$ & $208(81.3)$ & $48(18.8)$ & & $182(71.1)$ & $74(28.9)$ & & $122(47.7)$ & $134(52.3)$ & \\
\hline Present & $246(49.0)$ & $153(62.2)$ & $93(37.8)$ & & $163(66.3)$ & $83(33.7)$ & & 103 (41.9) & $143(58.1)$ & \\
\hline Neural invasion & & & & $<0.001$ & & & 0.571 & & & 0.086 \\
\hline Absent & $330(65.7)$ & $264(80.0)$ & $66(20.0)$ & & $224(67.9)$ & $106(32.1)$ & & 157 (47.6) & $173(52.4)$ & \\
\hline Present & $172(34.3)$ & $97(56.4)$ & $75(43.6)$ & & $121(70.3)$ & $51(29.7)$ & & $68(39.5)$ & $104(60.5)$ & \\
\hline Depth of invasion (pT) & & & & $<0.001$ & & & 0.221 & & & 0.156 \\
\hline $\mathrm{T} 1-\mathrm{T} 2$ & $295(58.8)$ & $239(81.0)$ & $56(19.0)$ & & $209(70.8)$ & $86(29.2)$ & & $140(47.5)$ & $155(52.5)$ & \\
\hline T3-T4 & $207(41.2)$ & $122(58.9)$ & $85(41.1)$ & & $136(65.7)$ & $71(34.3)$ & & $85(41.1)$ & $122(58.9)$ & \\
\hline Lymph node metastasis & & & & $<0.001$ & & & 0.809 & & & 0.459 \\
\hline No & $263(52.4)$ & $213(81.0)$ & $50(19.0)$ & & $182(69.2)$ & $81(30.8)$ & & $122(46.4)$ & $141(53.6)$ & \\
\hline $\mathrm{N}(+)$ & $239(47.6)$ & 148 (61.9) & $91(38.1)$ & & $163(68.2)$ & $76(31.8)$ & & $103(43.1)$ & $136(56.9)$ & \\
\hline pTNM stage & & & & $<0.001$ & & & 0.604 & & & 0.607 \\
\hline I-II & $334(66.5)$ & $262(78.4)$ & $72(21.6)$ & & $227(68.0)$ & $107(32.0)$ & & $147(44.0)$ & $187(56.0)$ & \\
\hline III-IV & $168(33.5)$ & $99(58.9)$ & $69(41.1)$ & & $118(70.2)$ & $50(29.8)$ & & $78(46.4)$ & $90(53.6)$ & \\
\hline Tumor multiplicity & & & & 0.264 & & & 0.186 & & & 0.739 \\
\hline No & $471(93.8)$ & $336(71.3)$ & $135(28.7)$ & & $327(69.4)$ & $144(30.6)$ & & $212(45.0)$ & $259(55.0)$ & \\
\hline Yes & $31(6.2)$ & $25(80.6)$ & $6(19.4)$ & & $18(58.1)$ & $13(41.9)$ & & 13(41.9) & $18(58.1)$ & \\
\hline HER2 status & & & & 0.992 & & & 0.022 & & & 0.186 \\
\hline Negative & $477(95.0)$ & $343(95.0)$ & $134(28.1)$ & & $333(96.5)$ & $144(30.2)$ & & 217 (96.4) & $260(54.5)$ & \\
\hline Positive & $25(5.0)$ & $18(72.0)$ & $7(28.0)$ & & $12(48.0)$ & $13(52.0)$ & & $8(32.0)$ & $17(68.0)$ & \\
\hline
\end{tabular}


Table 1 continued

\begin{tabular}{|c|c|c|c|c|c|c|c|c|c|c|}
\hline \multirow[t]{2}{*}{ Characteristics } & \multirow[t]{2}{*}{ Total $(\%)$} & \multicolumn{3}{|l|}{ NRG1 (\%) } & \multicolumn{3}{|l|}{ HER3 (\%) } & \multicolumn{3}{|c|}{ HER4 (cytoplasmic) (\%) } \\
\hline & & Negative & Positive & $P$ & Negative & Positive & $P$ & Negative & Positive & $P$ \\
\hline MSI status $(n=489)$ & & & & 0.336 & & & 0.215 & & & 0.762 \\
\hline MSS/MSI-L & $449(91.8)$ & $324(72.2)$ & $125(27.8)$ & & $312(69.5)$ & $137(30.5)$ & & $202(45.0)$ & $247(55.0)$ & \\
\hline MSI-H & $40(8.2)$ & $26(65.0)$ & $14(35.0)$ & & $24(60.0)$ & $16(40.0)$ & & $17(42.5)$ & $23(57.5)$ & \\
\hline EBV status $(\mathrm{n}=501)$ & & & & 0.332 & & & 0.454 & & & 0.192 \\
\hline Negative & $451(90.0)$ & $327(72.5)$ & $124(27.5)$ & & $312(69.2)$ & $139(30.8)$ & & $206(45.7)$ & $245(54.3)$ & \\
\hline Positive & $50(10.0)$ & $33(66.0)$ & $17(34.0)$ & & $32(64.0)$ & $18(36.0)$ & & $18(36.0)$ & $32(64.0)$ & \\
\hline
\end{tabular}

MSI microsatellite instability, EBV Epstein-Barr virus, NRG1 neuregulin 1, HER2 human epidermal growth factor 2, HER3 human epidermal growth factor receptor 3, HER4 human epidermal growth factor receptor 4

Table 2 Univariate and multivariate analysis for disease-free and disease-specific survival in gastric cancer

\begin{tabular}{|c|c|c|c|c|c|}
\hline \multirow[t]{2}{*}{ Variables } & \multirow[t]{2}{*}{ Category } & \multicolumn{2}{|l|}{ Disease-free survival } & \multicolumn{2}{|l|}{ Disease-specific survival } \\
\hline & & HR $(95 \% \mathrm{CI})$ & $P$ & $\mathrm{HR}(95 \% \mathrm{CI})$ & $P$ \\
\hline \multicolumn{6}{|l|}{ Univariate analysis } \\
\hline Age (years) & $>60$ vs. $\leq 60$ & $1.154(0.801-1.662)$ & 0.443 & $1.245(0.851-1.821)$ & 0.258 \\
\hline Tumor size & $>3$ vs. $\leq 3 \mathrm{~cm}$ & $10.163(4.469-23.110)$ & $<0.001$ & $11.309(4.610-27.741)$ & $<0.001$ \\
\hline Lauren classification & Non-intestinal vs. intestinal & $2.228(1.485-3.344)$ & $<0.001$ & $2.115(1.396-3.204)$ & $<0.001$ \\
\hline Ming classification & Infiltrative vs. expanding & $3.217(1.988-5.205)$ & $<0.001$ & $3.400(2.051-5.636)$ & $<0.001$ \\
\hline Vascular invasion & Present vs. absent & $6.078(4.137-8.932)$ & $<0.001$ & $6.364(4.279-9.464)$ & $<0.001$ \\
\hline Lymphatic invasion & Present vs. absent & $8.938(5.197-15.372)$ & $<0.001$ & $9.541(5.345-17.031)$ & $<0.001$ \\
\hline Neural invasion & Present vs. absent & $7.900(5.187-12.030)$ & $<0.001$ & 8.705 (5.566-13.614) & $<0.001$ \\
\hline pTNM stage & III, IV vs. I, II & $24.361(13.658-43.452)$ & $<0.001$ & $23.829(13.063-43.470)$ & $<0.001$ \\
\hline MSI status & MSI_H vs. MSS/MSI-L & $0.588(0.259-1.338)$ & 0.205 & $0.626(0.275-1.425)$ & 0.264 \\
\hline NRG1 & Positive vs. negative & $2.458(1.710-3.532)$ & $<0.001$ & $2.450(1.683-3.567)$ & $<0.001$ \\
\hline HER3 & Positive vs. negative & $1.227(0.842-1.788)$ & 0.288 & $1.150(0.776-1.703)$ & 0.487 \\
\hline HER4 & Positive vs. negative & $1.232(0.852-1.781)$ & 0.268 & $1.180(0.807-1.726)$ & 0.393 \\
\hline \multicolumn{6}{|l|}{ Multivariate analysis } \\
\hline Tumor size & $>3$ vs. $\leq 3 \mathrm{~cm}$ & $2.171(0.901-5.228)$ & 0.084 & $2.395(0.924-6.208)$ & 0.072 \\
\hline Lauren classification & Non-intestinal vs. intestinal & $0.810(0.531-1.236)$ & 0.328 & $0.775(0.503-1.194)$ & 0.248 \\
\hline Ming classification & Infiltrative vs. expanding & $0.894(0.528-1.514)$ & 0.677 & $0.975(0.562-1.691)$ & 0.928 \\
\hline Vascular invasion & Present vs. absent & $1.785(1.203-2.648)$ & 0.004 & $1.846(1.228-2.776)$ & 0.003 \\
\hline Lymphatic invasion & Present vs. absent & $1.911(1.044-3.499)$ & 0.036 & $2.086(1.110-3.919)$ & 0.022 \\
\hline Neural invasion & Present vs. absent & $1.450(0.890-2.363)$ & 0.136 & $1.655(1.008-2.717)$ & 0.046 \\
\hline pTNM stage & III, IV vs. I, II & $14.008(7.312-26.836)$ & $<0.001$ & $9.896(4.875-20.086)$ & $<0.001$ \\
\hline NRG1 & Positive vs. negative & $1.455(1.009-2.100)$ & 0.045 & $1.490(1.019-2.177)$ & 0.040 \\
\hline
\end{tabular}

NRG1 neuregulin 1, HER3 human epidermal growth factor receptor 3, HER4 human epidermal growth factor receptor 4, $H R$ hazard ratio, $C I$ confidence interval

$(P<0.001 ;$ kappa $=0.459$; Table 4). However, the two cases with NRG1 amplification were negative for NRG1 by IHC analysis, and NRG1 GCN gain was not observed in the majority of NRG1 IHC-positive cases $(65 / 113,57.5 \%)$. NRG1 GCN gain was significantly associated with diffuse or mixed type by the Lauren classification $(P=0.001)$, lymphatic invasion $(P=0.013)$, and lymph node metastasis $(P=0.013$; Supplementary material 1). By Kaplan-Meier analysis, patients with NRGl GCN gain had shorter DFS and DSS with borderline statistical significance ( $P=0.082$ and $P=0.078$, respectively; Fig. 2$)$, but Cox regression analysis indicated that $N R G 1$ GCN gain was not an independent prognostic factor $(P>0.05$, data not shown). 
Table 3 Correlation among expression of NRG1, HER3, and HER4

\begin{tabular}{|c|c|c|c|c|c|c|}
\hline & \multicolumn{3}{|l|}{ HER3 } & \multicolumn{3}{|l|}{ HER4 } \\
\hline & Negative & Positive & $P$ & Negative & Positive & $P$ \\
\hline NRG1 & & & 0.034 & & & $<0.001$ \\
\hline Negative & $258(71.5 \%)$ & $103(28.5 \%)$ & & $184(51.0 \%)$ & $177(49.0 \%)$ & \\
\hline Positive & $87(61.7 \%)$ & $54(38.3 \%)$ & & $41(29.1 \%)$ & $100(70.9 \%)$ & \\
\hline HER3 & & & & & & $<0.001$ \\
\hline Negative & & & & $182(52.8 \%)$ & $163(47.2 \%)$ & \\
\hline Positive & & & & $43(27.4 \%)$ & $114(72.6 \%)$ & \\
\hline
\end{tabular}

NRG1 neuregulin 1, HER3 human epidermal growth factor receptor 3, HER4 human epidermal growth factor receptor 4

\section{Correlation between HER3 membranous expression and clinicopathologic factors}

Positive expression of HER3 was predominantly observed in the cytoplasm, and 13 of 502 cases (2.6\%) also showed HER3 membranous expression (Supplementary material 2). GC cases with HER 3 membranous expression correlated with lymphatic invasion $(P=0.009)$, lymph node metastasis $(P=0.032)$, and mixed type according to the Lauren classification $(P=0.002)$, but did not correlate with other clinicopathologic factors including MSI and EBV status (all $P>0.05$, Supplementary material 1 ). GC patients with HER3 membranous expression had an unfavorable outcome for DFS $(P=0.018)$ and DSS $(P=0.015)$ by univariate analysis (Supplementary material 3). However, it was not an independent prognostic factor by multivariate analysis for DFS (HR 1.835; 95\% CI $1.798-4.218 ; P=0.153)$ and DSS (HR $1.744 ; 95 \%$ CI $0.757-4.016 ; P=0.191)$.

\section{Clinicopathologic significance of HER4 nuclear expression}

We next evaluated the clinical significance of HER4 nuclear expression in GC. HER4 nuclear expression was observed in $115(22.9 \%)$ of 502 GC cases (Supplementary material 2). HER4 nuclear expression was significantly associated with less aggressive clinicopathologic features, such as smaller tumor size, expanding tumor border, absence of lymphovascular and neural invasion, and early pathologic stage (all $P<0.05$ ). HER4 nuclear expression was also associated with intestinal type GC with borderline statistical significance $(P=0.052$; Supplementary material 1). In survival analysis, the HER4 nuclear expression group had superior DFS and DSS (both $P<0.001$; Supplementary material 3); however, in a multivariate hazard model, it no longer exhibited prognostic significance for either DFS or DSS (hazard ratio 1.088; 95\% confidence interval 0.561-2.111;
$P=0.803$ and Hazard ratio $0.901 ; 95 \%$ confidence interval $0.438-1.854 ; P=0.778$, respectively).

\section{Discussion}

To date, the clinicopathologic role of NRG1 in GC has been unclear; therefore, we investigated the clinicopathologic implications and prognostic value of NRG1 expression in GC specimens. NRG1 overexpression was observed in $28.1 \%$ of GC samples, and NRG1 status was strongly associated with aggressive clinicopathologic parameters, including larger tumor size, infiltrative tumor border, lymphovascular invasion, neural invasion, lymph node metastasis, and advanced pathologic stage. Additionally, the overexpression of NRG1 predicted poor prognosis in patients with GC. To the best of our knowledge, this is the first study to demonstrate the clinicopathologic significance of NRG1 expression in a large-scale study of GC.

NRG1, a member of the NRG family, acts by binding to HER 3 and HER4. HER3 is considered the major receptor for NRG1 [26, 27]. Recently, NRG1 has become the focus of research attention because of its overexpression in various cancers, including breast, urinary bladder, colorectal, prostate, and lung cancers [6]. In breast cancer, NRG1 overexpression was observed in approximately $30-80 \%$ of cases. In addition, NRG1 overexpression has been implicated in the activation of the HER3/HER2 signaling pathway, which mediates cancer cell proliferation, and other malignant features, including tumor invasion and metastasis [28-30]. Despite the increasing focus on NRG1 in various cancers, few studies have investigated the expression of NRG1 and its association with clinical outcome in GC. Han et al. [23] reported that NRG1 overexpression was significantly related to advanced pathologic stage, lymph node metastasis, and poor prognosis; however, there have been several conflicting reports on the prognostic significance of NRG1 overexpression in various cancers [31-33]. Our results indicate that NRG1 overexpression is strongly 

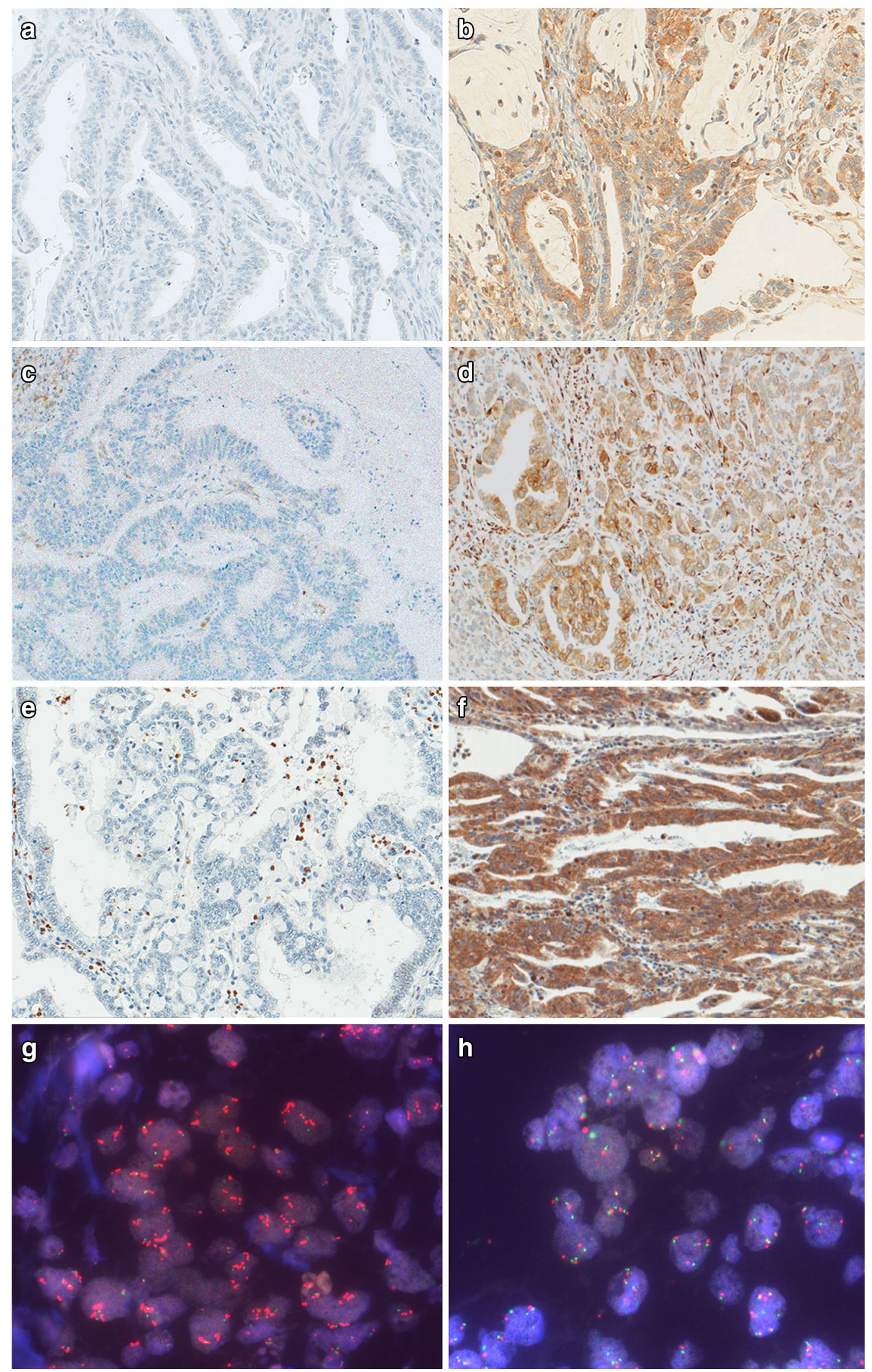
४Fig. 1 Representative images of NRG1, HER3, HER4 protein expression, and the NRG1 FISH assay in GC specimens. a NRG1 negative. b NRG1 positive. c HER3 negative. d HER3 positive. e HER4 negative. $\mathbf{f}$ HER4 positive. $\mathbf{g} N R G 1$ amplification. $\mathbf{h} N R G 1$ GCN gain

Table 4 Correlation between NRG1 immunohistochemistry and GCN status

\begin{tabular}{lrrrr}
\hline & \multicolumn{2}{l}{ NRG1 IHC } & & \multirow{2}{*}{$\kappa$} \\
\cline { 2 - 3 } & Negative & Positive & & \\
\hline NRG1 GCN & & & & \\
GCN non-gain & $266(80.4 \%)$ & $65(19.6 \%)$ & $<0.001$ & 0.459 \\
GCN gain & $9(15.8 \%)$ & $48(84.2 \%)$ & & \\
\hline
\end{tabular}

NRG1 neuregulin 1, IHC immunohistochemistry, GCN gene copy number, $\kappa$ Kappa coefficient

associated with unfavorable clinicopathologic features in GC. Moreover, we identified pronounced differences between outcomes in GC patients with or without NRG1 overexpression. Hence, the results of the present study suggest that NRG1 overexpression may be an independent poor prognostic factor in GC.

Because of the close relationship between NRG1 and HER3, NRG1 expression has been suggested as a predictive biomarker for HER3 inhibition [6, 11]. In addition, NRG1 can promote resistance to HER2-targeted therapy through activation of HER3 and PI3K/Akt signaling both in vivo and in vitro $[9,34,35]$. Furthermore, a combination of anti-HER2 treatment with administration of a HER3 inhibitor has been proposed as a promising therapeutic strategy to improve tumor regression [36]. Therefore, our NRG1 expression and GCN results provide basic information of potential use for the development of clinical trials of HER3 inhibitor therapy and combined HER2 and HER3 inhibitor therapy. The expression and genetic status of NRG1 may facilitate identification of a GC patient subgroup who could benefit from anti-HER3 treatment.

Previous studies demonstrated that HER3 was overexpressed in the cytoplasm or membrane of tumor cells, which predicted poor prognosis in GC $[15,37,38]$. However, in our result, patients with cytoplasmic and/or membranous expression of HER3 have suffered slightly shorter DFS and DSS, without statistical significance $(P>0.05)$, and HER3 overexpression did not correlate with lymph node metastasis or stage $(P>0.05)$. By subgroup analysis HER3 overexpression was associated with unfavorable prognosis in diffuse type $\mathrm{GC}(P=0.025$, data not shown), but not in intestinal type $(P>0.05)$. Furthermore, GCs with membranous expression of HER3 showed significantly worse outcomes $(P=0.018)$. Therefore, the survival analyses of HER 3 expression may be affected by histologic subtypes and intracellular sublocalization (cytoplasmic vs. membranous). It may be additionally influenced by the sample size, race, ethnicity, antibody sources, and immunostaining protocol. However, our results showed that HER3 overexpression was significantly associated with HER2 positivity $(P=0.022)$ and the intestinal type of the Lauren classification $(P<0.001)$, consistent with most previous studies [39, 40].

Recent studies have also highlighted the clinical implications of HER4, since its expression is detected in various cancers [18, 41, 42]. Notably, HER4 has two conflicting roles in cancer. It can both inhibit and promote cell proliferation, depending on the localization of different HER4 isoforms generated by alternative splicing [18-20]. Alternative splicing of the HER4 gene leads to the production of two intracytoplasmic isoforms, CYT1 and CYT2. Compared with CYT2, translocation into the nucleus by CYT1 is less efficient. CYT1 also can induce the PI3K/Akt pathway, leading to increased cell proliferation and inhibition of cell differentiation [19, 43]. Depending on the presence of these isoforms, HER4 may show different intracellular localizations and varying clinical significance in malignancies. Previous studies on HER4 expression in GC failed to demonstrate a significant association with patients survival $[15,39]$, and little is known about the function of NRG1 in relation to the subcellular distribution of HER4 in GC. In a review of breast cancer studies, while HER4 cytoplasmic expression was favorably associated with patient survival, the significance of HER4 expression localized to the nucleus with regard to survival was uncertain [18]. In the current analysis, we evaluated HER4 nuclear and cytoplasmic expression independently in GC, according to the localization of immunostaining. We found that HER4 nuclear expression was tightly associated with favorable clinicopathologic features and better survival rates in GC; however, HER4 cytoplasmic expression failed to show a significant association with these parameters, in contrast to the reported results for this protein in breast cancer. Moreover, NRG1 expression was tightly related to cytoplasmic expression of HER4 and exhibited an inverse association with HER4 nuclear expression, with borderline statistical significance (data not shown). Considering the conflicting role of HER4 in cancer, our findings suggested that HER4 nuclear rather than cytoplasmic expression might be related to favorable clinical characteristics.

Our results demonstrate that NRGI amplification is a relatively rare event $(0.5 \%)$ in GC. This is consistent with the findings of a previous study, which demonstrated that $N R G 1$ amplification is infrequent in GC [23]; however, alterations in NRG1 GCN have not previously been investigated in GC. Despite the lack of acknowledged 
Fig. 2 Kaplan-Meier survival estimates according to NRG1, HER 3 and HER4 protein expression, and NRG1 GCN status. DFS and DSS according to $\mathbf{a}, \mathbf{b}$ NRG1, c, d HER3 and $\mathbf{e}$, f HER4 expression, and $\mathbf{g}$,

h $N R G 1 \mathrm{GCN}$ status
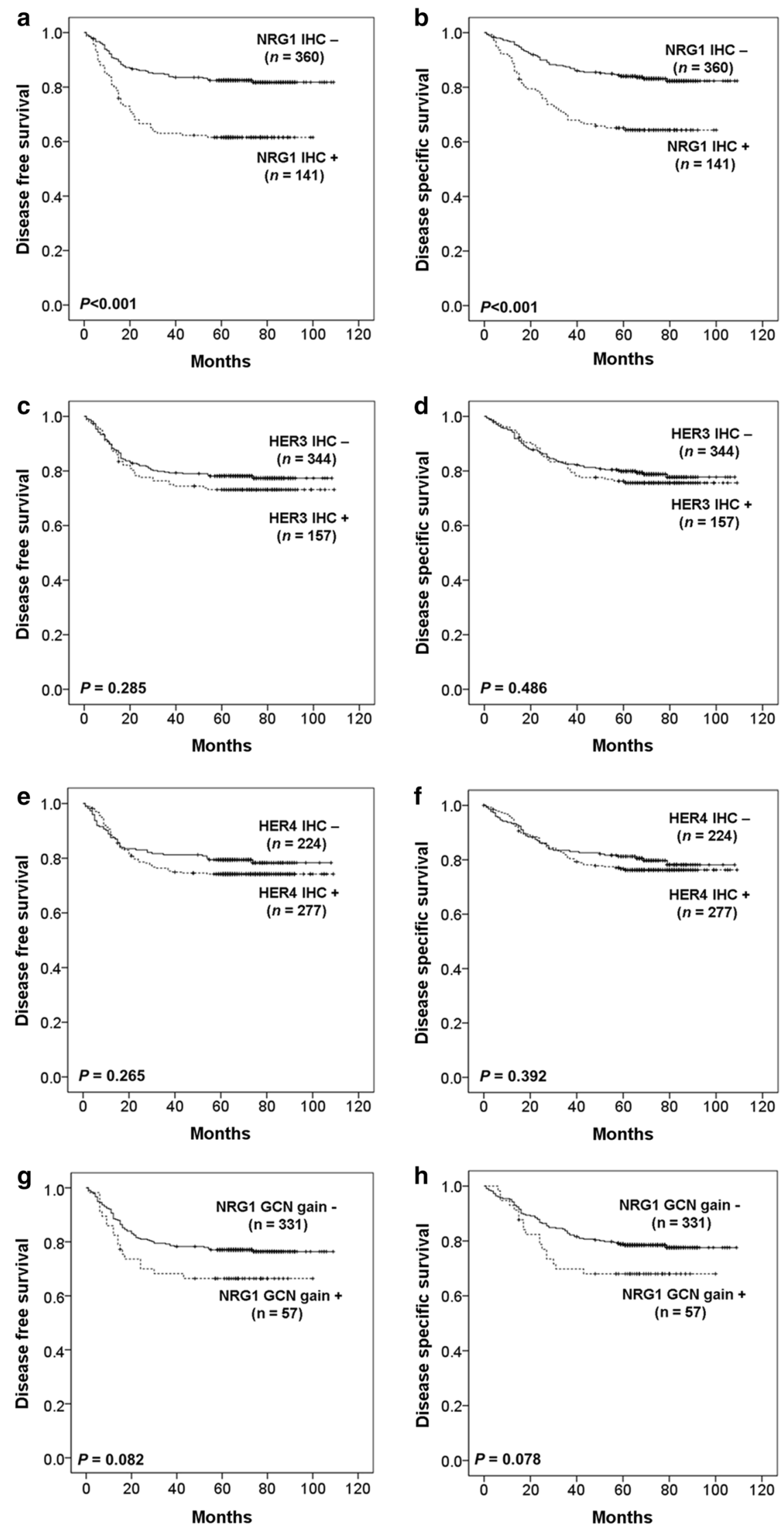
consensus criteria for GCN gain, our results revealed that this phenomenon was observed with relatively low frequency (14.7\%). Additionally, we compared NRG1 protein expression and gene status. A significant discrepancy between NRG1 GCN alteration and protein expression was identified, with cancer cells exhibiting NRG1 amplification found to be negative for NRG1 immunostaining. One possible explanation for this discrepancy is that NRG1 may be overexpressed through mechanisms other than GCN alteration or gene amplification.

Our study has some limitations, including sampling bias of TMA slides, the use of a single institute retrospective cohort, and a lack of inclusion of patients receiving HER3 inhibitor therapy. Therefore, further comprehensive studies and clinical trials are necessary to clarify the usefulness of NRG1 for the identification of cases where anti-HER3 treatment would be appropriate.

In conclusion, we evaluated the clinical significance of NRG1 and its receptors, including HER3 and HER4, in a large cohort of patients with GC. NRG1 was frequently overexpressed, and its expression was highly correlated with those of HER3 and HER4 in GC. We also identified a strong correlation between high levels of NRG1 protein expression and increased NRGI GCN. Moreover, overexpression of this protein was significantly associated with aggressive behavior of GC including poor prognosis. However, the expression of HER3 and HER4 was not significantly associated with patient outcome. These results suggest that NRG1 overexpression may predict poor clinical outcome and that targeting NRG1 represents a therapeutic opportunity in GC.

Acknowledgements This study was funded through a grant of the Korea Health Technology R\&D Project through the Korea Health Industry Development Institute (KHIDI), funded by the Ministry of Health and Welfare, Republic of Korea (Grant No. HI14C1813).

\section{Compliance with ethical standards}

Conflict of interest The authors declare that they have no conflict of interest.

Ethics statement All procedures in this study were conducted in accordance with the ethical standards of the responsible institutional committee on human experimentation and with the Helsinki Declaration of 1964 and later versions. This study was approved by the institutional review board (IRB) of Seoul National University Bundang Hospital (IRB no. B-1407-260-305). The need to acquire written informed consents was waived by the IRB on condition of anonymization.

\section{References}

1. Jung KW, Won YJ, Oh CM, Kong HJ, Cho H, Lee JK, et al. Prediction of cancer incidence and mortality in Korea, 2016. Cancer Res Treat. 2016;48(2):451-7. doi:10.4143/crt.2016.092 (Epub 2016/04/02).
2. De Vita F, Giuliani F, Silvestris N, Rossetti S, Pizzolorusso A, Santabarbara G, et al. Current status of targeted therapies in advanced gastric cancer. Expert Opin Ther Targets. 2012;2012(16 Suppl 2):S29-34. doi:10.1517/14728222.2011. 652616 (Epub 2012/03/27).

3. Yi JH, Kang JH, Hwang IG, Ahn HK, Baek HJ, Lee SI, et al. A retrospective analysis for patients with HER2-positive gastric cancer who were treated with Trastuzumab-based chemotherapy: in the perspectives of ethnicity and histology. Cancer Res Treat. 2016;48(2):553-60. doi:10.4143/crt.2015.155 (Epub 2015/09/ 02).

4. Matsuoka T, Yashiro M. Recent advances in the HER2 targeted therapy of gastric cancer. World J Clin Cases. 2015;3(1):42-51. doi:10.12998/wjcc.v3.i1.42 (Epub 2015/01/23).

5. Shimoyama S. Unraveling trastuzumab and lapatinib inefficiency in gastric cancer: future steps (review). Mol Clin Oncol. 2014;2(2):175-81. doi:10.3892/mco.2013.218 (Epub 2014/03/ 22).

6. Ocana A, Diez-Gonzalez L, Esparis-Ogando A, Montero JC, Amir E, Pandiella A. Neuregulin expression in solid tumors: prognostic value and predictive role to anti-HER3 therapies. Oncotarget. 2016. doi:10.18632/oncotarget.8648 (Epub 2016/04/ 14).

7. Montero JC, Rodriguez-Barrueco R, Ocana A, Diaz-Rodriguez E, Esparis-Ogando A, Pandiella A. Neuregulins and cancer. Clin Cancer Res. 2008;14(11):3237-41. doi:10.1158/1078-0432.ccr07-5133 (Epub 2008/06/04).

8. Poovassery JS, Kang JC, Kim D, Ober RJ, Ward ES. Antibody targeting of HER2/HER3 signaling overcomes heregulin-induced resistance to $\mathrm{PI} 3 \mathrm{~K}$ inhibition in prostate cancer. Int $\mathrm{J}$ Cancer. 2015;137(2):267-77. doi:10.1002/ijc.29378 (Epub 2014/12/05).

9. Xia W, Petricoin EF 3rd, Zhao S, Liu L, Osada T, Cheng Q, et al. An heregulin-EGFR-HER3 autocrine signaling axis can mediate acquired lapatinib resistance in HER2 + breast cancer models. Breast Cancer Res. 2013;15(5):R85. doi:10.1186/bcr3480 (Epub 2013/09/21).

10. Tao JJ, Castel P, Radosevic-Robin N, Elkabets M, Auricchio N, Aceto N, et al. Antagonism of EGFR and HER3 enhances the response to inhibitors of the PI3K-Akt pathway in triple-negative breast cancer. Sci Signal. 2014;7(318):ra29. doi:10.1126/sci signal.2005125 (Epub 2014/03/29).

11. Meetze K, Vincent S, Tyler S, Mazsa EK, Delpero AR, Bottega $S$, et al. Neuregulin 1 expression is a predictive biomarker for response to AV-203, an ERBB3 inhibitory antibody, in human tumor models. Clin Cancer Res. 2015;21(5):1106-14. doi:10. 1158/1078-0432.ccr-14-2407 (Epub 2014/12/30).

12. Jiang N, Saba NF, Chen ZG. Advances in Targeting HER3 as an Anticancer Therapy. Chemother Res Pract. 2012;2012:817304. doi:10.1155/2012/817304 (Epub 2012/12/01).

13. Baselga J, Swain SM. Novel anticancer targets: revisiting ERBB2 and discovering ERBB3. Nat Rev Cancer. 2009;9(7):463-75. doi:10.1038/nrc2656 (Epub 2009/06/19).

14. Sithanandam G, Anderson LM. The ERBB3 receptor in cancer and cancer gene therapy. Cancer Gene Ther. 2008;15(7):413-48. doi:10.1038/cgt.2008.15 (Epub 2008/04/12).

15. Hayashi M, Inokuchi M, Takagi Y, Yamada H, Kojima K, Kumagai J, et al. High expression of HER3 is associated with a decreased survival in gastric cancer. Clin Cancer Res. 2008;14(23):7843-9. doi:10.1158/1078-0432.ccr-08-1064 (Epub 2008/12/03).

16. Ema A, Yamashita K, Ushiku H, Kojo K, Minatani N, Kikuchi $\mathrm{M}$, et al. Immunohistochemical analysis of RTKs expression identified HER3 as a prognostic indicator of gastric cancer. Cancer Sci. 2014;105(12):1591-600. doi:10.1111/cas.12556 (Epub 2014/12/03). 
17. Cao GD, Chen K, Xiong MM, Chen B. HER3, but Not HER4, Plays an Essential Role in the Clinicopathology and Prognosis of Gastric Cancer: A Meta-Analysis. PLoS One. 2016;11(8):e0161219. doi:10.1371/journal.pone.0161219 (Epub 2016/08/19).

18. Wang J, Yin J, Yang Q, Ding F, Chen X, Li B, et al. Human epidermal growth factor receptor 4 (HER4) is a favorable prognostic marker of breast cancer: a systematic review and metaanalysis. Oncotarget. 2016; doi:10.18632/oncotarget.12485 (Epub 2016/10/14).

19. Fujiwara S, Hung M, Yamamoto-Ibusuk CM, Yamamoto $Y$, Yamamoto S, Tomiguchi M, et al. The localization of HER4 intracellular domain and expression of its alternately-spliced isoforms have prognostic significance in ER + HER2 - breast cancer. Oncotarget. 2014;5(11):3919-30. doi:10.18632/onco target.2002 (Epub 2014/07/09).

20. Mohd Nafi SN, Generali D, Kramer-Marek G, Gijsen M, Strina C, Cappelletti M, et al. Nuclear HER4 mediates acquired resistance to trastuzumab and is associated with poor outcome in HER2 positive breast cancer. Oncotarget. 2014;5(15):5934-49. doi:10.18632/oncotarget.1904 (Epub 2014/08/26).

21. Edge SBBD, Compton CC, Fritz AG, Greene FL, Trotti A, editors. AJCC cancer staging manual. 7th ed. New York: Springer; 2010.

22. Ryu HS. Park do J, Kim HH, Kim WH, Lee HS. Combination of epithelial-mesenchymal transition and cancer stem cell-like phenotypes has independent prognostic value in gastric cancer. Hum Pathol. 2012;43(4):520-8. doi:10.1016/j.humpath.2011.07. 003 (Epub 2011/10/25).

23. Han ME, Kim HJ, Shin DH, Hwang SH, Kang CD, Oh SO. Overexpression of NRG1 promotes progression of gastric cancer by regulating the self-renewal of cancer stem cells. J Gastroenterol. 2015;50(6):645-56. doi:10.1007/s00535-014-1008-1 (Epub 2014/11/09).

24. Higaki E, Kuwata T, Nagatsuma AK, Nishida Y, Kinoshita T, Aizawa M, et al. Gene copy number gain of EGFR is a poor prognostic biomarker in gastric cancer: evaluation of 855 patients with bright-field dual in situ hybridization (DISH) method. Gastric Cancer. 2016;19(1):63-73. doi:10.1007/s10120-0140449-9 (Epub 2014/12/10).

25. Seo AN, Kwak Y, Kim DW, Kang SB, Choe G, Kim WH, et al. HER2 status in colorectal cancer: its clinical significance and the relationship between HER2 gene amplification and expression. PLoS One. 2014;9(5):e98528. doi:10.1371/journal.pone.0098528 (Epub 2014/06/01).

26. Holbro T, Civenni G, Hynes NE. The ErbB receptors and their role in cancer progression. Exp Cell Res. 2003;284(1):99-110 (Epub 2003/03/22).

27. Carraway KL 3rd, Sliwkowski MX, Akita R, Platko JV, Guy PM, Nuijens A, et al. The erbB3 gene product is a receptor for heregulin. J Biol Chem. 1994;269(19):14303-6 (Epub 1994/05/ 13).

28. Stove $\mathrm{C}$, Bracke M. Roles for neuregulins in human cancer. Clin Exp Metastasis. 2004;21(8):665-84 (Epub 2005/07/23).

29. Atlas E, Cardillo M, Mehmi I, Zahedkargaran H, Tang C, Lupu R. Heregulin is sufficient for the promotion of tumorigenicity and metastasis of breast cancer cells in vivo. Mol Cancer Res. 2003;1(3):165-75 (Epub 2003/01/31).

30. Breuleux M. Role of heregulin in human cancer. Cell Mol Life Sci. 2007;64(18):2358-77. doi:10.1007/s00018-007-7120-0 (Epub 2007/05/29).
31. Esteva FJ, Hortobagyi GN, Sahin AA, Smith TL, Chin DM, Liang SY, et al. Expression of erbB/HER receptors, heregulin and P38 in primary breast cancer using quantitative immunohistochemistry. Pathol Oncol Res. 2001;7(3):171-7 (Epub 2001/11/ 03).

32. Mitsui K, Yonezawa M, Tatsuguchi A, Shinji S, Gudis K, Tanaka S, et al. Localization of phosphorylated ErbB1-4 and heregulin in colorectal cancer. BMC Cancer. 2014;14:863. doi:10.1186/14712407-14-863 (Epub 2014/11/25).

33. Forster JA, Paul AB, Harnden P, Knowles MA. Expression of NRG1 and its receptors in human bladder cancer. Br J Cancer. 2011;104(7):1135-43. doi:10.1038/bjc.2011.39 (Epub 2011/03/ 03).

34. Sato Y, Yashiro M, Takakura N. Heregulin induces resistance to lapatinib-mediated growth inhibition of HER2-amplified cancer cells. Cancer Sci. 2013;104(12):1618-25. doi:10.1111/cas. 12290 (Epub 2013/10/12).

35. Wilson TR, Lee DY, Berry L, Shames DS, Settleman J. Neuregulin-1-mediated autocrine signaling underlies sensitivity to HER2 kinase inhibitors in a subset of human cancers. Cancer Cell. 2011;20(2):158-72. doi:10.1016/j.ccr.2011.07.011 (Epub 2011/08/16).

36. Kang JC, Poovassery JS, Bansal P, You S, Manjarres IM, Ober $\mathrm{RJ}$, et al. Engineering multivalent antibodies to target heregulininduced HER3 signaling in breast cancer cells. MAbs. 2014;6(2):340-53. doi:10.4161/mabs.27658 (Epub 2014/02/05).

37. Tang D, Liu CY, Shen D, Fan S, Su X, Ye P, et al. Assessment and prognostic analysis of EGFR, HER2, and HER3 protein expression in surgically resected gastric adenocarcinomas. Onco Targets Ther. 2015;8:7-14. doi:10.2147/ott.s70922 (Epub 2015/01/08).

38. Wu WK, Tse TT, Sung JJ, Li ZJ, Yu L, Cho CH. Expression of ErbB receptors and their cognate ligands in gastric and colon cancer cell lines. Anticancer Res. 2009;29(1):229-34 (Epub 2009/04/01).

39. He XX, Ding L, Lin Y, Shu M, Wen JM, Xue L. Protein expression of HER2, 3, 4 in gastric cancer: correlation with clinical features and survival. J Clin Pathol. 2015;68(5):374-80. doi:10.1136/jclinpath-2014-202657 (Epub 2015/03/04).

40. Jacome AA, Wohnrath DR, Scapulatempo Neto C, Carneseca EC, Serrano SV, Viana LS, et al. Prognostic value of epidermal growth factor receptors in gastric cancer: a survival analysis by Weibull model incorporating long-term survivors. Gastric Cancer. 2014;17(1):76-86. doi:10.1007/s10120-013-0236-z (Epub 2013/03/05).

41. Haskins JW, Nguyen DX, Stern DF. Neuregulin 1-activated ERBB4 interacts with YAP to induce Hippo pathway target genes and promote cell migration. Sci Signal. 2014;7(355):ra116. doi:10.1126/scisignal.2005770 (Epub 2014/12/11).

42. Okazaki S, Nakatani F, Masuko K, Tsuchihashi K, Ueda S, Masuko T, et al. Development of an ErbB4 monoclonal antibody that blocks neuregulin-1-induced ErbB4 activation in cancer cells. Biochem Biophys Res Commun. 2016;470(1):239-44. doi:10.1016/j.bbrc.2016.01.045 (Epub 2016/01/19).

43. Sundvall M, Peri L, Maatta JA, Tvorogov D, Paatero I, Savisalo $\mathrm{M}$, et al. Differential nuclear localization and kinase activity of alternative ErbB4 intracellular domains. Oncogene. 2007;26(48):6905-14. doi:10.1038/sj.onc.1210501 (Epub 2007/05/09). 